\title{
Éditorial
}

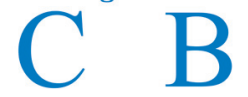

\section{Direction Vancouver via Helsinki !}

\author{
Jacky Samson*
}

La difficulté n'est pas de comprendre les idées nouvelles mais d'échapper aux idées anciennes. John Maynard Keynes

Au fil des années, la revue Médecine Buccale Chirurgie Buccale a pris du corps et de la tenue. Pendant presque 20 ans, elle a accompagné la spécialité de Chirurgie orale qui était en gestation. Maintenant, on peut considérer qu'elle a atteint la taille adulte mais il lui manque encore la reconnaissance internationale pour être l'égal des grandes revues de spécialités. Pour obtenir cette reconnaissance internationale, entre autres les référencements Scopus et Medline, nous devons respecter la Déclarartion d'Helsinki et les recommandations du Groupe de Vancouver.

Tout le monde a entendu parler de la déclaration d'Helsinki mais cela reste souvent un peu flou. Après la Deuxième Guerre mondiale, l'Association Médicale Mondiale (AMM) a succédé à l'Association Professionnelle Internationale des Médecins, créée en 1926, et qui avait cessé ses activités au début de la guerre. A l'initiative de médecins anglais, il y eut une conférence à Londres en septembre 1946 qui a abouti à la création officielle de l'AMM, avec l'assentiment des anciens dirigeants de la précédente association. La première Assemblée générale de l'AMM eut lieu en septembre 1947 à Paris et, depuis cette date, l'AMM organise chaque année une réunion, l'Assemblée Médicale Mondiale, qui regroupe des médecins venus de tous les horizons. Le siège social de l'AMM, initialement situé à New York (Etats Unis), a été transféré en 1975 à FerneyVoltaire (France).

En réaction aux crimes contre l'humanité commis par les médecins nazis pendant la Deuxiéme Guerre mondiale au nom de la recherche médicale, l'AMM s'intéressa en raison du contexte d'abord à l'éthique. Elle proposa de remplacer le Serment d'Hippocrate, un peu désuet et devenu trop souvent une formalité, par un engagement actualisé et plus formel des médecins envers le but humanitaire de la médecine. Cet engagement, connu sous le nom de Déclaration de Genève, a été adopté lors de la $2^{\mathrm{e}}$ Assemblée générale qui s'est tenue à Genève (Suisse) en septembre 1948. La Déclaration de Genève a été amendée par la suite à plusieurs reprises (1968, 1983,
1994, 2005 et 2006) afin d'être toujours le reflet de la médecine contemporaine, et dans l'espoir qu'elle soit adoptée par tous.

Après son rapport sur les « Crimes de guerre et la médecine » remis lors de la $2^{\mathrm{e}}$ Assemblée générale, l'AMM a décidé de préparer un Code international d'Ethique médicale qui fut adopté lors de la $3^{\mathrm{e}}$ Assemblée générale. L'AMM a décidé de poursuivre son activité en s'intéressant principalement à l'expérimentation sur des êtres humains. Ces travaux ont abouti à la Déclaration d'Helsinki [1] qui fut adoptée lors de la $18^{\mathrm{e}}$ Assemblée générale en juin 1964 à Helsinki (Finlande) ; elle fut amendée à diverses reprises (1975, 1983, 1989, 1996, 2000, 2002, 2004 et 2008). Dans sa dernière version, le premier paragraphe de cette Déclaration définit clairement le champ d'application : "L'AMM a élaboré la Déclaration d'Helsinki comme un énoncé de principes éthiques applicables à la recherche médicale impliquant des êtres humains, y compris la recherche sur du matériel biologique et sur des données identifiables. ». Les différentes versions ont été rassemblées par Philippe Amiel [3]. Cette lecture est sans aucun doute fastidieuse mais on peut avoir une vue synoptique des différents amendements et appréhender la démarche de l'AMM en consultant le travail de Xavier Aurey [4]. Dès le premier amendement en 1975, l'AMM a introduit la notion de protection des animaux et de l'environement ; dans la dernière version, cela se traduit ainsi : «Le bien être des animaux utilisés dans la recherche doit être respecté. » (principe B. 12 fin) et « Une prudence particulière s'impose dans la conduite de recherches susceptibles de nuire à l'environnement. » (principe B. 13). En conséquence, "L'investigateur doit être attentif aux dispositions éthiques, légales et réglementaires applicables à la recherche sur les sujets humains dans son propre pays ainsi qu'aux règles internationales applicables. Aucune disposition nationale d'ordre éthique, légal et réglementaire ne doit conduire à affaiblir ou supprimer les mesures protectrices énoncées dans la présente déclaration. ».

\footnotetext{
*Correspondance : jacky.samson@unige.ch
} 
En 1978, les éditeurs des cinq principaux périodiques médicaux - 3 sont américains : Journal of the American Medical Association, New England Journal of Medicine et Annals of Internal Medicine ; 2 sont anglais : The Lancet et British Medical Journal -, (les « big five »), se sont réunis à Vancouver (Canada) afin d'établir des directives pour préparer et rédiger les articles soumis à leur revue. Au fil du temps, le groupe s'est un peu ouvert et il est devenu l'International Committee of Medical Journal Editors (ICMJE) ou « Groupe de Vancouver ». Les recommandations ont pris progressivement la forme d'un guide de bonnes pratiques intitulé « Uniform Requirements for Manuscripts Submitted to Biomedical Journals », souvent abrégé sous la forme de URM. La dernière version, datée d'avril 2010, comporte trois sections: les considérations éthiques relatives à la conduite et à la présentation des recherches, les problèmes d'édition et de rédaction liés à la publication dans des revues biomédicales, et la préparation et la soumission d'un manuscrit. La version officielle est disponible sur le site www.icmje.org. Cependant, de temps à autre, l'ICMJE doit reconnaître d'autres structures ou adopte parfois une attitude jugée excessive. Par exemple, en 1997, après avoir listé les 35 modèles de présentation des références existant dans les versions antérieures, il a reconnu la préséance de la National Library of Medicine [5]. Autre exemple, plus récemment, il a proposé un formulaire de déclaration de conflits d'intérêt que certains ont considéré comme quasi inquisitorial. Mais dans l'ensemble, avec le temps, les mesures préconisées par l'ICMJE ont eu un effet bénéfique incontestable sur les publications médicales.

La HAS, en réalisant une traduction en français de ce guide qu'elle a intitulé «Exigences uniformes pour les manuscrits soumis aux revues biomédicales : rédaction et édition de la publication médicale »[6], nous invite indirectement à les adopter. Prochainement le site de la revue Médecine Buccale Chirurgie Buccale sera profondément remanié pour respecter les recommandations de l'ICMJE. En conséquence, vous verrez apparaître une nouvelle version des conseils aux auteurs, la description du processus d'évaluation des articles et des formulaires pour la déclaration des conflits d'intérêt et pour le transfert des droits d'auteur. Ainsi qu'un site pour la soumission en ligne des articles. Tout ceci devrait favoriser nos démarches pour le référencement. Enfin, nous demanderons à être répertorié sur le site www.icmje.org comme revue respectant les exigences uniformes.

\section{Références}

1. http://www.wma.net/fr/30publications/10policies/b3/17c_fr.pdf (consulté le 22 mars 2013).

2. Amiel Ph. http://descobayesetdeshommes.fr/Docs/032-Helsinki Versions1964-2008.pdf (consulté le 22 mars 2013).

3. Aurey X. http://www.fondamentaux.org/2011/09/27/tableausur-la-declaration-dhelsinki-et-ses-revisions/ (consulté le 22 mars 2013).

4. http://fr.wikipedia.org/wiki/Déclaration_d'Helsinki (consulté le 22 mars 2013).

5. Patrias K. Citing Medicine: the NLM style guide for authors, editors, and publishers. 2nd ed. Wendling $D$, technical editor. Bethesda (MD): National Library of Medicine (US), 2007. http://www.ncbi.nlm.nih.gov/books/NBK7256/ (consulté le 22 mars 2013).

6. http://www.icmje.org/french.pdf (consulté le 22 mars 2013). 